\title{
Model of cell damage in single session radiation treatments based on continuous-time Markov chains
}

\author{
Roukaya Keinj ${ }^{*}$ Magalie Thomassin ${ }^{* *}$ Thierry Bastogne ${ }^{* * *}$ \\ Pierre Vallois ${ }^{* * *}$ \\ * Université Joseph Fourier, Laboratoire Jean Kuntzmann - Tour \\ IRMA, Equipe Projet INRIA MOISE, B.P. 53, 38041 Grenoble Cedex \\ 9, France, (e-mail: roukaya.keinj@imag.fr) \\ ** University of Lorraine, Centre de Recherche en Automatique de \\ Nancy, CNRS UMR 7039 BP 70239, F-54506 Vandouvre-lès-Nancy \\ Cedex, France, (e-mail: magalie.thomassin@univ-lorraine.fr) \\ *** University of Lorraine, INRIA-BIGS \& Centre de Recherche en \\ Automatique de Nancy, CNRS UMR 7039 \\ **** University of Lorraine, INRIA-BIGS 8 Institut de Mathématiques \\ Elie Cartan, CNRS UMR 7502
}

\begin{abstract}
A new continuous-time statistical model for modelling the effect of radiotherapy treatment is proposed. In contrast with classical models, it takes in account heterogeneity of cell damage and repair. In this paper, a bi-scale continuous-time model is derived to describe tumor and normal tissue lifespans. We show that the cell lifespan can be described by a continuous-time Markov model. The tumor control probabilities and normal tissue complication probabilities are expressed. The model has been implemented into Matlab and numerical simulations have emphasised the effects of the model parameters on the model output.
\end{abstract}

Keywords: cancer, radiotherapy, continuous-time Markov chain, cell damage heterogeneity, lifespan, optimisation treatment.

\section{INTRODUCTION}

One of the common therapies used to treat cancer consists in using ionizing (X-ray) or non-ionizing (light) radiations to cause a variety of possible lesions in cells (Curtis, 1986). Mathematical modeling may help to quantify the effects of radiation-based treatments on cell populations. It can be used to predict tumor growth and cancer spread, but also allows to determine the effectiveness of a specific treatment.

In a previous paper (Keinj et al., 2011), the authors studied the cell response to treatment and the tumor growth by considering Markov chains. The discrete times correspond to the successive times of application of radiation dose fractions. In this paper, we consider continuoustime processes to model the tumor response during the continuous application of radiation dose in treatments such as permanent brachytherapy in which the duration of the total dose may vary from a few weeks to a month, or photodynamic therapy, where the duration of the light dose may vary from seconds to tens of minutes. The proposed bi-scale model reproduces both damages in cell and tumor scales. At the cell scale, the damage level is described by a discrete-state variable. We show that the probability to be in a given state follows a system of linear differential equations. We also establish the lifespan model of a cancer cell and the one of a tumor.
Two probabilities are generally involved in the design of a treatment plan in radiotherapy : (i) the tumor control probability (TCP) and (ii) the normal tissue complication probability (NTCP). The TCP is the probability that all cancer cells are dead in the irradiated region, see (Zaider and Minerbo, 2000; Dawson and Hillen, 2006). The NTCP is another probability that measures the sensitivity of normal tissue to radiations. These two probabilities are strongly related to the cell and tumor lifespans. In this paper, we use this relationship to give new expressions of TCP and NTCP suited to single session treatments.

This paper is organized as follows. In Section 2, we develop a continuous-time Markov chain model of a cancer cell response to radiation treatment. In Section 3, we express the lifespans of tumors and normal tissues and we give a detailed calculation of their properties. In section 4, we formulate the new expressions of TCP and NTCP.

\section{MARKOV MODEL OF THE CELL STATE}

In this section, the behavior of a single cell during the treatment is considered.

\subsection{Reminders related to the multinomial model}

Let us briefly recall the main features of the model which has been developed in (Keinj et al., 2011, 2012) in the context of fractioned radiotherapy treatment: 


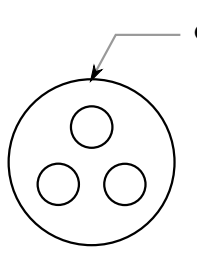

cell
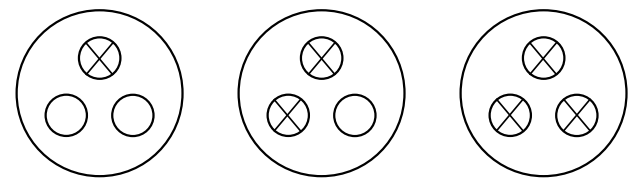

$Z_{k}=0$ $\bigcirc$ active target $\bigotimes$ inactive target

$Z_{k}=2$
$Z_{k}=3$

Fig. 1. Damage state $Z_{k}$ of a cell with $m=3$ targets

- each cell has $m$ targets,

- each target can be deactivated by a single hit with a radiation particle with probability $q$,

- death occurs when the $m$ targets are inactive,

- between two consecutive doses a target can be reactivated (repair process) with probability $r$, while the cell is still alive.

Figure 1 shows the case of a 3-target cell and the corresponding cell states.

Let $Z_{k}$ be the random number of deactivated targets in the cell at time $k$, i.e. after the $k^{\text {th }}$ dose fraction. It is supposed that $\left(Z_{k}\right)_{k \geqslant 0}$ is a Markov chain which takes its values in $\{0,1, \ldots, m\}$. Denote $\boldsymbol{\Pi}$ the associated transition matrix. The effect of the treatment and the repair process are taken into account by supposing that $\boldsymbol{\Pi}=\mathbf{P R}$ where:

$$
\begin{aligned}
& \mathbf{P}(i, j)=\left\{\begin{array}{cc}
\left(\begin{array}{c}
m-i \\
j-i
\end{array}\right) q^{j-i}(1-q)^{m-j} & i \leqslant j \\
0 & j \geqslant i
\end{array}\right. \\
& \mathbf{R}(i, j)=\left\{\begin{array}{cc}
\left(\begin{array}{c}
i \\
i-j
\end{array}\right) r^{i-j}(1-r)^{j} & j \leqslant i<m \\
0 & i<j,
\end{array}\right.
\end{aligned}
$$

where by convention the first row and column are labeled 0 .

Note that $q$ and hence $\alpha$, depends on the applied radiation dose $u$. Polynomial models, like the Linear Quadratic model (Fowler, 1989), can be used to describe this relationship between $\alpha$ and $u$.

\subsection{Toward the model of continuous-time Markov chain}

Suppose that $h=\Delta t$ is a small interval of time. For a given cell, let $Z_{k h}$ be the number of deactivated targets at time $t=k h$. It is assumed that $\left(Z_{k h}\right)_{k \geqslant 0}$ is a Markov chain with transition $\boldsymbol{\Pi}_{h}$ depending on the three parameters $m, q_{h}$ and $r_{h}$. The parameter $q_{h}$ is the probability that an active target is deactivated in any small interval $[k h,(k+1) h]$. Similarly, $r_{h}$ is the probability that an inactive target in a living cell is repaired during the interval $[k h,(k+1) h]$. Since $h$ is small, it seems natural to assume that both $q_{h}$ and $r_{h}$ are small: between two consecutive times $t$ and $t+\Delta t$, the cell stays mainly in the same state: changes are possible but with a low probability. More precisely, we suppose:

$$
\begin{aligned}
& q_{h}=\alpha h+o(h) \\
& r_{h}=\rho h+o(h)
\end{aligned}
$$

with $\alpha, \rho>0$ and $\lim _{h \rightarrow 0} \frac{o(h)}{h}=0$.

From equations (1) and (3), it is easy to show that:

$$
\begin{cases}\mathbf{P}_{h}(i, i)=1-\alpha(m-i) h+o(h) & \\ \mathbf{P}_{h}(i, i+1)=\alpha(m-i) h+o(h) & i<m \\ \mathbf{P}_{h}(i, j)=o\left(h^{2}\right) & j>i+1 \\ \mathbf{P}_{h}(i, j)=0 & j<i\end{cases}
$$

and from (2) and (4), we obtain:

$$
\begin{cases}\mathbf{R}_{h}(i, i)=1-i \rho h+o(h) & \\ \mathbf{R}_{h}(i, i-1)=i \rho h+o(h) & i>0 \\ \mathbf{R}_{h}(i, j)=o\left(h^{2}\right) & j<i-1 \\ \mathbf{R}_{h}(i, j)=0 & j>i\end{cases}
$$

So, using classical analysis, we can deduce that the transition probabilities $\boldsymbol{\Pi}_{h}(i, j)=\mathbf{P}_{h} \mathbf{R}_{h}$ of $\left(Z_{k h}\right)_{k \geqslant 0}$ is given by the following identities:

$$
\begin{aligned}
& \Pi_{h}(i, j)=\operatorname{Pr}\left(Z_{k h+h}=j \mid Z_{k h}=i\right)= \\
& \left\{\begin{array}{lll}
\alpha(m-i) h+o(h) & j=i+1,0 \leqslant i \leqslant m-1 \\
1-[\alpha(m-i)+i \rho] h+o(h) & j=i, \quad 0 \leqslant i \leqslant m-1 \\
i \rho h+o(h) & j=i-1, \quad 1 \leqslant i \leqslant m-1 \\
1 & j=i=m \\
0 & \text { else. }
\end{array}\right.
\end{aligned}
$$

\subsection{Definition of the continuous-time Markov chain $\left(Z_{t}\right)$}

Let $Z_{t}$ be the number of deactivated targets in a cell at time $t$. We suppose that $\left(Z_{t}\right)_{t \geqslant 0}$ is a continuous-time Markov chain. Previous analysis suggests to set:

$$
\begin{gathered}
\operatorname{Pr}\left(Z_{t+h}=j \mid Z_{t}=i\right)=\boldsymbol{\pi}_{h}(i, j) \\
\text { if } t \geqslant 0, h>0,0 \leqslant i, j \leqslant m \text { and } \\
\boldsymbol{\pi}_{h}(i, j)= \begin{cases}1+\mathbf{Q}(i, j) h+o(h) & \text { if } j=i \\
\mathbf{Q}(i, j) h+o(h) & \text { if } j \neq i\end{cases}
\end{gathered}
$$

where $\mathbf{Q}(i, j)$ is defined by:

$$
\begin{aligned}
& \mathbf{Q}(i, j)= \\
& \begin{cases}\alpha(m-i) & j=i+1,0 \leqslant i \leqslant m-1 \\
-[\alpha(m-i)+i \rho] & j=i, \quad 0 \leqslant i \leqslant m-1 \\
i \rho & j=i-1,1 \leqslant i \leqslant m-1 \\
0 & \text { else. }\end{cases}
\end{aligned}
$$

We deduce easily from (9):

$$
\begin{cases}\mathbf{Q}(i, j) \leqslant 0 & \text { if } i=j \\ \mathbf{Q}(i, j) \geqslant 0 & \text { if } i \neq j \\ \sum_{j=0}^{m} \mathbf{Q}(i, j)=0 & 0 \leqslant i \leqslant m\end{cases}
$$

As a consequence, Theorem 2.8.2 in (Norris, 1997) and (10) imply existence of a Markov chain $\left(Z_{t}\right)_{t \geqslant 0}$ with generator matrix $\mathbf{Q}$ and $\{0,1, \ldots, m\}$-valued.

\subsection{Probability distribution of $Z_{t}$}

Given the generator matrix $\mathbf{Q}$, we can determine the transition probability matrix $\boldsymbol{\Pi}(t)$ with entries $\boldsymbol{\Pi}_{t}(i, j)$. Each entries is the probability for the cell to being in the state $j$ at time $t \geqslant 0$ when its initial state is $Z_{0}=i$ :

$$
\boldsymbol{\pi}_{i, j}(t) \triangleq \operatorname{Pr}\left(Z_{t}=j \mid Z_{0}=i\right) \quad 0 \leqslant i, j \leqslant m .
$$


Here, we are mainly interested in the case where the cell is initially in state $Z_{0}=0$. This leads to introduce:

$$
P_{j}(t) \triangleq \operatorname{Pr}\left(Z_{t}=j \mid Z_{0}=0\right)=\pi_{0, j}(t) \quad j \in 0, \ldots, m .
$$

Recall that the set of matrices $\Pi(t) \triangleq\left(\pi_{i, j}(t)\right)_{(0 \leqslant i, j \leqslant m)}$ satisfies the following linear differential equation:

$$
\boldsymbol{\Pi}^{\prime}(t)=\boldsymbol{\Pi}(t) \mathbf{Q} \text {. }
$$

Consequently, the functions $P_{0}(t), \ldots, P_{0}(t)$ solve the following system of $m+1$ linear differential equations:

$$
P_{j}^{\prime}(t)=(\boldsymbol{\Pi}(t) \mathbf{Q})(0, j)=\sum_{k=0}^{m} q_{k, j} P_{k}(t) \quad 0 \leqslant j \leqslant m
$$

with the initial conditions:

$$
\left(P_{0}(0), P_{1}(0), \ldots, P_{m}(0)\right)=(1,0, \ldots, 0)
$$

Using the explicit form of $\mathbf{Q}$ (if (9)), system (14) can be simplified as:

$$
\begin{aligned}
& P_{j}^{\prime}(t)=\alpha(m-(j-1)) P_{j-1}(t) \\
& \quad-((m-j) \alpha+j \rho) P_{j}(t)+(j+1) \rho \mathbb{1}_{\{j \leqslant m-2\}} P_{j+1}(t)
\end{aligned}
$$

if $1 \leqslant j \leqslant m-1$ and otherwise:

$$
P_{j}^{\prime}(t)= \begin{cases}-m \alpha P_{0}(t)+\rho P_{1}(t) & j=0 \\ \alpha P_{m-1}(t) & j=m .\end{cases}
$$

Proposition 1. The transpose matrix $\mathbf{Q}^{T}$ of $\mathbf{Q}$ which is diagonalizable and has $m+1$ distinct eigenvalues $\lambda_{i}$ which satisfy

$$
-m \max (\alpha, \rho) \leqslant \lambda_{m}<\ldots<\lambda_{1}<\lambda_{0}=0 .
$$

The proof is given in (Keinj, 2011).

Although the matrix $\mathbf{Q}$ is a simple function of parameters $m, \alpha$ and $\rho$, it is not possible to determine explicitly the eigenvalues of $\mathbf{Q}$. However, for given values of $m, \alpha$ and $\rho$, the eigenvalues $\lambda_{1}, \ldots, \lambda_{m}$ and the associated eigenvectors can be numerically calculated.

Proposition 2. The system (14) with initial conditions $\left(P_{0}(0), P_{1}(0), \ldots, P_{m}(0)\right)=(1,0, \ldots, 0)$ has a unique solution. Moreover, there exists $m+1$ vectors in $\mathbb{R}^{m+1}$, $M_{0}, \ldots, M_{m}$ such that:

$$
P(t)=\sum_{j=0}^{m} M_{j} e^{\lambda_{j} t}
$$

where $P(t)=\left(P_{0}(t), \ldots, P_{m}(t)\right)^{T}$ and $\lambda_{0}=0, \lambda_{1}, \ldots, \lambda_{m}$ are the eigenvalues of $\mathbf{Q}^{T}$ (cf. Proposition 1$)$.

Proof. The system (14) is equivalent to $P^{\prime}(t)=\mathbf{Q}^{T} P(t)$. Therefore $P(t)=e^{t \mathbf{Q}^{T}} P(0)$. Since $\mathbf{Q}^{T}$ is diagonalizable, (19) follows directly.

It is important to emphasize that once the values of $\alpha$, $\rho$ and $m$ are known, our model permits to evaluate the probability of a cell to be in a state (from 0 to $m$ ) at the time $t$. In particular, the model allows one to determine the probability that a cell is dead at time $t$ since $P_{m}(t)=$ $\operatorname{Pr}\left(Z_{t}=m \mid Z_{0}=0\right)$. This property will play an important role in the following section.

We have implanted the probabilities $P_{j}(t)$ of being in a state $j$ for $m=3$ into the computing environment Matlab. From $P_{m}(t)$, it is possible to determine $t_{0}$ such that $\forall t \geqslant t_{0}$

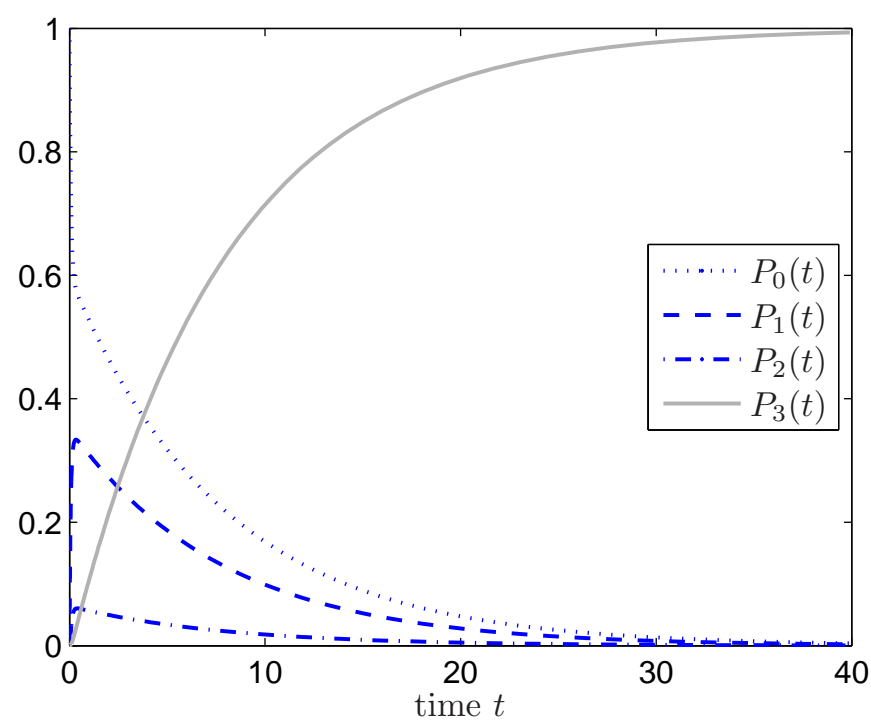

Fig. 2. Probability $P_{j}(t)$ of being in the state $k \in$ $\{0, \ldots, m\}$ at time $t \geqslant 0$ with parameters $m=3$, $\alpha=2$ and $\rho=10$.

$P_{m}(t) \geqslant 95 \%$. Then, we can say that the cell is dead after $t_{0}$ with a risk of not more than $5 \%$.

Fig. 2 shows the probability $P_{j}(t)$ with parameters $m=3$, $\alpha=2$ and $\rho=10$. We can see that $P_{3}(t)$ is superior to 0.95 from $t_{0}=25$. The table 1 give $t_{0}$ for different values of $m, \alpha$ and $\rho$.

Table 1 . Some values of $t_{0}$ for different values of $m, \alpha$ and $\rho$

\begin{tabular}{|l||c|c|c||c|c|c|}
\hline \multicolumn{1}{|c||}{$t_{0}$} & \multicolumn{3}{c||}{$m=3$} & \multicolumn{3}{c|}{$m=4$} \\
\cline { 2 - 7 } & $\alpha=2$ & $\alpha=3$ & $\alpha=8$ & $\alpha=2$ & $\alpha=3$ & $\alpha=8$ \\
\hline \hline$\rho=5$ & 10 & 4.5 & 0.87 & 26 & 9 & 1.22 \\
$\rho=10$ & 25 & 9.5 & 1.32 & 105 & 29.5 & 2.34 \\
$\rho=15$ & 44 & 16 & 1.85 & 274 & 70 & 4 \\
\hline
\end{tabular}

In the following section, we will focus on the properties of the lifespan of a cell and of a set of cells (i.e. for a tumor).

\section{LIFESPAN ESTIMATION}

\subsection{Lifespan of a cancer cell}

Let $T$ be a random variable denoting the lifespan of a single cell (with $m$ targets). In other words, $T$ is the first time $t$ when the cell is in state $m$ (death state):

$$
T=\inf \left\{t>0, Z_{t}=m\right\} \text {. }
$$

In this part, we will give the cumulative distribution function of $T$.

Proposition 3. Suppose that $Z_{0}=0$, then the cumulative distribution function of $T$ is given by:

$$
\operatorname{Pr}(T \leqslant t)=P_{m}(t)=1+\sum_{j=1}^{m} M_{j, m} e^{\lambda_{j} t}
$$

where the coefficients $M_{j, m}$ are constant.

Proof. As the initial state of the cell is $Z_{0}=0$ and the state $m$ is an absorbing state, we have:

$\operatorname{Pr}(T \leqslant t)=\operatorname{Pr}\left(Z_{t}=m \mid Z_{0}=0\right)=P_{m}(t) \quad \forall t \geqslant 0$. 
Relation (19) implies that $P_{m}(t)$ is a linear combination of decreasing exponential functions:

$$
\operatorname{Pr}(T \leqslant t)=\sum_{j=0}^{m} M_{j, m} e^{\lambda_{j} t} .
$$

Since $\operatorname{Pr}(T \leqslant t)$ is the cumulative distribution function of $T$ :

$$
\operatorname{Pr}(T \leqslant t)=\sum_{j=0}^{m} M_{j, m} e^{\lambda_{j} t} \underset{t \rightarrow \infty}{\longrightarrow} 1 .
$$

Recall that $\lambda_{m}<\ldots<\lambda_{1}<\lambda_{0}=0$, therefore (24) implies $M_{0, m}=1$ and $(21)$.

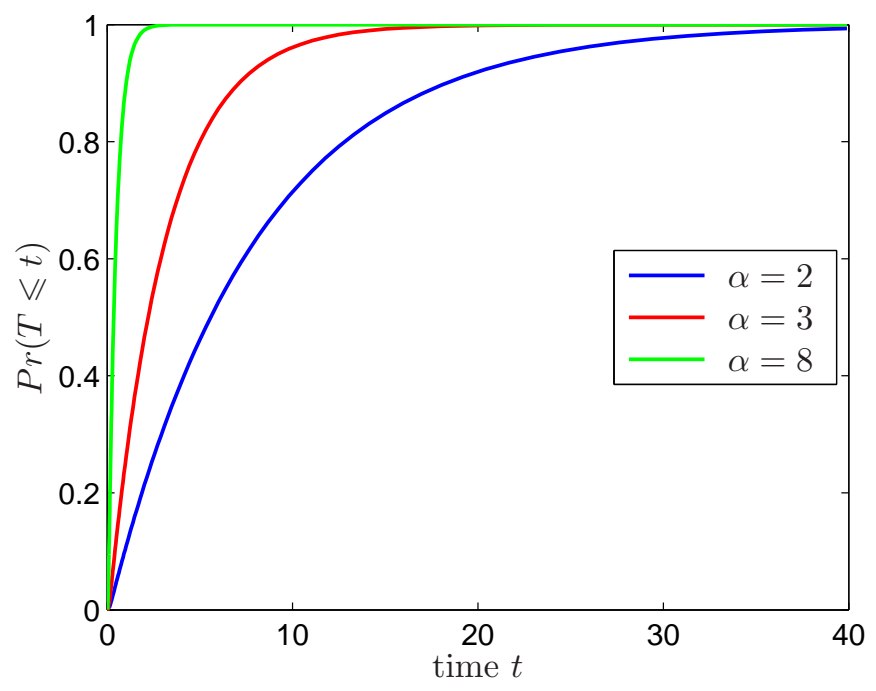

Fig. 3. Influence of $\alpha$ on the distribution function of $T$ for $m=3, \rho=10$ and $\alpha=2,3,8$.

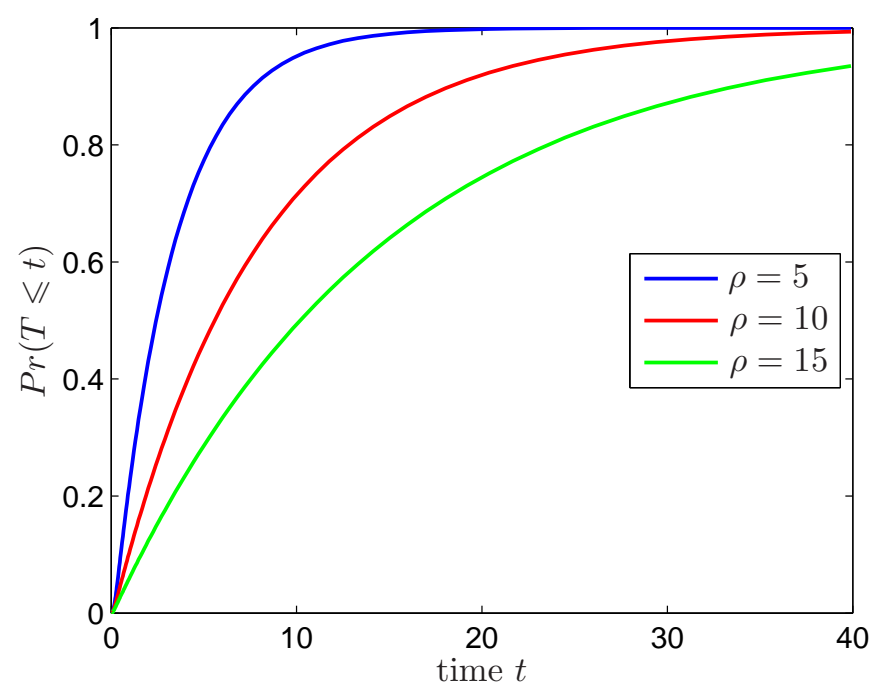

Fig. 4. Influence of $\rho$ on the distribution function of $T$ for $m=3, \alpha=2$ and $\rho=5,10,15$.

Fig. 3 presents the graph of the probability distribution function (pdf) of $T$ when $m=3, \rho=10$ and $\alpha$ varies in $\{2,3,8\}$. Let $F_{\alpha}$ be the related pdf. Note that $F_{\alpha}(t)=$ $P_{m}(t)$ and $P_{m}(t)$ has been determined by solving (16) and (17). We observe that $F_{8}(t) \leqslant F_{3}(t) \leqslant F_{2}(t)$. These inequalities intuitively means that $T_{(2)} \leqslant T_{(3)} \leqslant T_{(8)}$ where $T_{\left(\alpha_{0}\right)}$ is the lifespan of the cell when $\alpha=\alpha_{0}$. This is coherent with intuition : more the treatment is efficient, i.e. more $\alpha$ is large, more the lifespan of the cell decreases.

Similarly, we also consider the case $m=3$ and $\alpha=2$ and $\rho=5,10,15$. The relative position of the 3 curves reveals that roughly speaking $T$ increases when the cell repair, i.e. $\rho$ is large.

One important feature of our model is to allow explicit calculation which can be easily evaluated with Matlab. Although our model is naive, we recover expected answers.

Proposition 4. The cumulative distribution function of $T$ can be asymptotically approached by an exponential distribution with parameter $\lambda=-\lambda_{1}>0$ where $\lambda_{1}$ is the highest negative eigenvalue of $\mathbf{Q}^{T}$ :

$$
1-\operatorname{Pr}(T \leqslant t) \sim \beta e^{-\lambda t} \quad(t \rightarrow+\infty)
$$

where $\beta>0$.

The proof of this proposition is given in (Keinj, 2011).

Let $F$ be the pdf of $T$. Roughly speaking, identity (25) implies that $F(t)$ is approximatively equal to $1-e^{-\lambda t}$ for large $t$. However nothing can be for intermediate $t$. Therefore, it is interesting to compare $F(t)$ with $1-e^{-\lambda t}$ for any $t$. We choose $m=3, \rho=10$ and $\alpha$ in $\{2,3,8\}$. We observe in Fig. 5 that for $t>0,4$ there are no main difference between the pdf of $T$ and its exponential approximation.

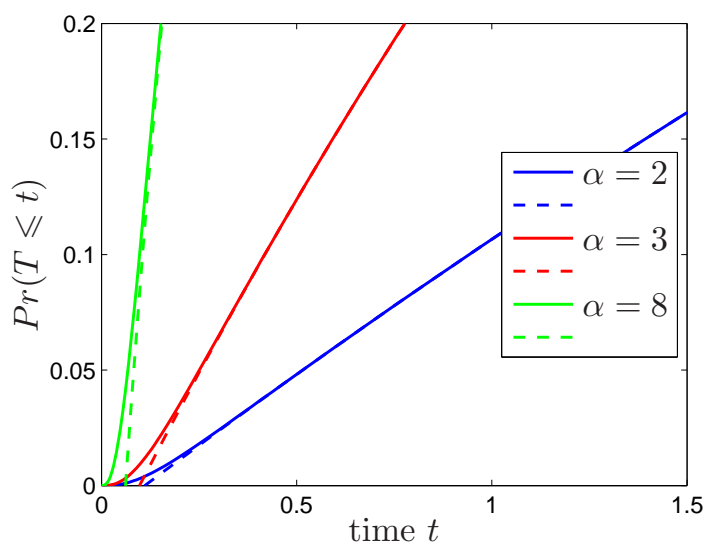

Fig. 5. Pdf of $T$ (solid line) and its approximation (dashed line) $1-\beta e^{-\lambda t}$ for $m=3, \rho=10$ and $\alpha=2,3,8$.

\subsection{Lifespan of a tumor}

We assume a tumor contains initially $n_{0}$ cells. Let us mention an important limitation to our model: we are not able to take into account proliferation of cells. For each cell $j$, we define its lifespan $T^{(j)}$ :

$$
T^{(j)}=\inf \left\{t>0, Z_{t}^{(j)}=m\right\}
$$

where $Z_{t}^{(j)}$ is the number of deactivated targets of the cell $j$ at time $t$. It follows that the lifespan of the tumor can be defined as the maximum of lifespans of the $n_{0}$ cells:

$$
L_{n_{0}}=\max \left\{T^{(1)}, T^{(2)}, \ldots, T^{\left(n_{0}\right)}\right\} .
$$

Assume that all the cells have the same behavior dynamics and are independent of each other. As a consequence, the variables $T^{(1)}, \ldots, T^{\left(n_{0}\right)}$ are i.i.d. and have the same 
distribution than $T$. Then the cumulative distribution function of $L_{n_{0}}$ is:

$$
\begin{aligned}
\operatorname{Pr}\left(L_{n_{0}} \leqslant t\right) & =\operatorname{Pr}\left(T^{(1)} \leqslant t\right) \times \ldots \times \operatorname{Pr}\left(T^{\left(n_{0}\right)} \leqslant t\right) \\
& =(\operatorname{Pr}(T \leqslant t))^{n_{0}}=\left(P_{m}(t)\right)^{n_{0}} .
\end{aligned}
$$

We consider tumors with a large initial number of cells. It has been proved in (Keinj et al., 2011) that for large values of $n_{0}$,

$$
\operatorname{Pr}\left(\lambda L_{n_{0}}-\ln \left(\beta n_{0}\right) \leqslant t\right) \approx e^{-e^{-t}} \quad t \in \mathbb{R} .
$$

Moreover, it has been demonstrated that the mean and the variance of $L_{n_{0}}$ has the following asymptotic behavior:

$$
\begin{aligned}
E\left(L_{n_{0}}\right) & \approx \frac{1}{\lambda} \ln \left(n_{0}\right)+\frac{\gamma+\ln (\beta)}{\lambda} \\
\operatorname{var}\left(L_{n_{0}}\right) & \approx \frac{\pi^{2}}{6 \lambda^{2}}
\end{aligned}
$$

where $\gamma$ is the Euler constant $\left(\gamma=-\Gamma^{\prime}(1), \Gamma(t)\right.$ being the gamma function).

\subsection{Lifespan of a normal tissue}

The main undesirable effect due to radiotherapy is the irradiation of normal tissue. Normal organs and tissues have different responses to radiations due to their distinct architecture. Niemierko and Goitein (Niemierko and Goitein, 1991) proposed a division of normal tissues into three different architectures: serial architecture (e.g. nerves or spinal cord), parallel architecture (e.g. kidney, liver or lung) and graduated response (e.g. skin or mucous membranes). We restrict ourselves to the parallel architecture. It is supposed that organs are composed of functional subunits (FSUs) and that organ function is compromised when the number of dead cells in the normal tissue is larger than a given threshold number $\bar{n}$ (reserve capacity of the tissue).

We now adapt the approach developed in (Keinj et al., 2012) to continuous-time. Let us consider a single normal cell that contains $\bar{m}$ targets. Let $\bar{Z}_{t}$ be the random variable denoting the state of a normal cell at time $t$ and $\bar{T}$ its lifespan. Similarly to the case of a cancer cell, we assume that $\left\{\bar{Z}_{t}\right\}_{t \geqslant 0}$ is a continuous-time Markov chain defined by the matrix $\overline{\mathbf{Q}}$, similar as $\mathbf{Q}$ in (9) with parameters $\bar{\alpha}$ and $\bar{\rho}$. Thus, for large $t$, the distribution function of $\bar{T}$ can be approximated:

$$
1-\operatorname{Pr}(\bar{T} \leqslant t) \sim \bar{\beta} e^{-\bar{\lambda} t} \quad(t \rightarrow+\infty)
$$

where $-\bar{\lambda}$ is the highest negative eigenvalue of $\overline{\mathbf{Q}}^{T}$ and $\bar{\beta}>0$.

Now, consider a normal tissue with initially $\bar{n}_{0}$ cells and with a reserve capacity $\bar{n}-1$ (functional tolerance). For each cell $j$ we define its lifespan:

$$
\bar{T}^{(j)}=\inf \left\{t>0, \bar{Z}_{t}^{(j)}=\bar{m}\right\},
$$

where $\bar{Z}_{t}^{(j)}$ is the number of deactivated targets among the $\bar{m}$ targets of the cell $j$. Let us rearrange these random variables into a nondecreasing sequence:

$$
\bar{T}^{\left(1: \bar{n}_{0}\right)} \leqslant \bar{T}^{\left(2: \bar{n}_{0}\right)} \leqslant \ldots \leqslant \bar{T}^{\left(\bar{n}_{0}: \bar{n}_{0}\right)}
$$

This sequence is the order statistic of $\bar{T}^{(1)}, \bar{T}^{(2)}, \ldots, \bar{T}^{\left(\bar{n}_{0}\right)}$. $\bar{T}^{\left(l: \bar{n}_{0}\right)}$ is called the $l^{\text {th }}$ order statistic and is the time when the $l^{\text {th }}$ cell died. According to our assumptions, the normal tissue is seriously damaged at the random time $L_{\bar{n}, \bar{n}_{0}}=\bar{T}^{\left(\bar{n}: \bar{n}_{0}\right)}$.
Let $\bar{X}_{t}$ be the number of dead normal cells among the $\bar{n}_{0}$ at time $t$. It is clear that:

$$
\operatorname{Pr}\left(L_{\bar{n}, \bar{n}_{0}} \leqslant t\right)=\operatorname{Pr}\left(\bar{X}_{t} \geqslant \bar{n}\right) .
$$

As a normal cell is died at time $t$ with a probability $\operatorname{Pr}(\bar{T} \leqslant \mathrm{t})=\bar{P}_{\bar{m}}(t)$ and all the cells are supposed to be independent:

$$
\bar{X}_{t} \sim \mathcal{B}\left(\bar{n}_{0}, \bar{P}_{\bar{m}}(t)\right)
$$

and

$$
\operatorname{Pr}\left(\bar{X}_{t} \geqslant \bar{n}\right)=\sum_{i=\bar{n}}^{\bar{n}_{0}}\left(\begin{array}{c}
\bar{n}_{0} \\
i
\end{array}\right)\left(\bar{P}_{\bar{m}}(t)\right)^{i}\left(1-\bar{P}_{\bar{m}}(t)\right)^{\bar{n}_{0}-i}
$$

Next, in (Keinj, 2011), it has been shown that for large value of $\bar{n}_{0}$,

$$
\operatorname{Pr}\left(L_{\bar{n}, \bar{n}_{0}} \leqslant t\right) \approx 1-e^{-\bar{n}_{0}(\bar{\alpha} t)^{\bar{m}}} \sum_{k=0}^{\bar{n}-1} \frac{\bar{n}_{0}^{k}(\bar{\alpha} t)^{\bar{m} k}}{k !}
$$

\section{TUMOR CONTROL PROBABILITY AND NORMAL TISSUE COMPLICATION PROBABILITY}

The TCP is the probability that all cancer cells are dead in the irradiated region and the NTCP measures the sensitivity of the adjacent normal tissue for a given radiation treatment schedule. In (Keinj et al., 2011), a strategy has been developped to both maximize the TCP and keep the NTCP lower than a given tolerance threshold.

\subsection{Tumor Control Probability}

The TCP at time $t$ is the probability that the tumor is dead at this time. As a result,

$$
\mathrm{TCP}(t)=\operatorname{Pr}\left(L_{n_{0}} \leqslant t\right) .
$$

When $n_{0}$ is large,

$$
\operatorname{TCP}(t) \approx e^{-\beta n_{0} e^{-\lambda t}} .
$$

It is clear that $(41)$ implies that $\operatorname{TCP}(t) \approx 0$, since $n_{0}$ is large and $t$ is fixed. Obviously, it is a very negative answer, which says that the treatment fails with probability close to 1 ! On way to have $\operatorname{TCP}(t) \approx 1$ when $n_{0}$ is large is to suppose that the parameter $\alpha$ is large and depends on $n_{0}$. In the discrete case (i.e. the model developed in (Keinj et al., 2011, 2012)), it has been proved that when $q$ is an increasing function of $n_{0}$, we can have $\operatorname{TCP}(t) \approx 1$.

\subsection{Normal Tissue Complication Probability}

The NTCP at time $t$ is the probability that a complication appears in the adjacent normal tissue. Therefore:

$$
\operatorname{NTCP}(t)=\operatorname{Pr}\left(L_{\bar{n}, \bar{n}_{0}} \leqslant t\right)
$$

If $n_{0}$ is large,

$$
\operatorname{NTCP}(t) \approx 1-e^{-\bar{n}_{0}(\bar{\alpha} t)^{\bar{m}}} \sum_{k=0}^{\bar{n}-1} \frac{\bar{n}_{0}^{k}(\bar{\alpha} t)^{\bar{m} k}}{k !}
$$

Note that (43) is valid with $\bar{n}$ fixed. As a result, $\operatorname{NTCP}(t)$ is close to 1 as $n_{0}$ is large. This actually means that, with a probability near 1 , all the $\bar{n}$ normal cells are damaged! This problem is similar to the one related to $\operatorname{TCP}(t)$. 


\section{CONCLUSION}

In this paper, a simple bi-scale continuous-time model based on Markov chains is derived to describe tumor and normal tissue lifespans. The proposed model takes into account the heterogeneity of cell damages and the repair mechanisms and allows to obtain exact formulas which depend only on 3 variables $m$ (the target number of a cell), $\rho$ and $\alpha$ (the probabilities of deactivating or activating a target in a small time interval). These parameters could be estimated from data of cell lifespans. A collaboration with a cancer treatment center is envisaged to obtain data and study the validity of the proposed model.

A possible perspective for this work is to develop a model which takes into account cell proliferation and normal dead. Moreover, it may also encompass current knowledge about biological effects of treatments. Anyhow, the resulting model will be certainly complex (requiring a large number of parameters). Thus its estimation will be difficult due to the small number of available biological data.

Finally, the expressions of TCP/NTCP and their discussions in the last section have raised some questions. A solution could be to consider that the parameter $\alpha$ is a time-decreasing function. However, in order to avoid model complication, it would be wiser to consider that $\alpha$ is constant over a small time interval which makes the derived expressions still valid.

\section{REFERENCES}

Curtis, S.B. (1986). Lethal and potentially lethal lesions induced by radiation - a unified repair model. Radiat. Res, 106, 252-279.

Dawson, A. and Hillen, T. (2006). Derivation of the tumour control probability (tcp) from a cell cycle model. Computational and Mathematical Methods in Medicine, $7(2-3), 121-141$.

Feinendegen, L., Hahnfeldt, P., Schadt, E.E., Stumpf, M., and Voit, E.O. (2008). Systems biology and its potential role in radiobiology. Radiat Environ Biophys, 47, 5-23.

Fowler, J. (1989). The linear-quadratic formula and progress in fractionated radiotherapy. Br. J Radiol, 62, 679-694.

Hillen, T., de Vries, G., Gong, J., and Finlay, C. (2010). From cell population models to tumor control probability: including cell cycle effects. In Acta Oncologica.

Keinj, R. (2011). Modélisation de la croissance d'une tumeur après traitement par radiothérapie. $\mathrm{Ph} . \mathrm{D}$. thesis, University of Lorraine, Nancy, France.

Keinj, R., Bastogne, T., and Vallois, P. (2011). Multinomial model-based formulations of TCP and NTCP for radiotherapy treatment planning. Journal of Theoretical Biology, 279(1), 55-62.

Keinj, R., Bastogne, T., and Vallois, P. (2012). Tumor growth modeling based on cell and tumor lifespans. Journal of Theoretical Biology. Submitted.

Kirkby, N.F., Burnet, N.G., and Faraday, D.B.F. (2002). Mathematical modelling of the response of tumour cells to radiotherapy. Nuclear Instruments and Methods in Physics Research. Section B: Beam Interactions with Materials and Atoms, 188, 210-215.
Niemierko, A. and Goitein, M. (1991). Calculation of normal tissue complication probability and dose-volume histogram reduction schemes for tissues with a critical element architecture. Radiotherapy and Oncology, 20(3), $166-176$.

Norris, J.R. (1997). Markov Chains. Cambridge Series in Statistical and Probabilistic Mathematics. Cambridge University Press, Cambridge, UK.

Sachs, R.K., Hlatky, L.R., and Hahnfeldt, P. (2001). Simple ODE models of tumor growth and anti-angiogenic or radiation treatment. Mathematical and Computer Modelling, 33, 1297-1305.

Zaider, M. and Minerbo, G.N. (2000). Tumour control probability: a formulation applicable to any temporal protocol of dose delivery. Physics in Medicine and Biology, 45(2), 279-293. URL http://stacks.iop.org/0031-9155/45/279. 